\title{
Pain intensity and functional limitation are not related with medical image findings in patients with shoulder pain*
}

\author{
A intensidade da dor e a limitação funcional não estão relacionadas com os achados em \\ imagens de pacientes com dor no ombro
}

Francine Aparecida Coelho de Oliveira ${ }^{1}$, Renato Santos de Almeida², Wagner Teixeira dos Santos ${ }^{3}$, Leandro Alberto Calazans Nogueira $^{4}$

${ }^{*}$ Recebido do Centro Universitário Augusto Motta, Rio de Janeiro, RJ, Brasil.

DOI 10.5935/1806-0013.20140044

\section{ABSTRACT}

BACKGROUND AND OBJECTIVES: Shoulder pain limits patients' ability to perform daily life activities, as well as to carry out professional activities. This study aimed at evaluating the relationship between pain intensity, incapacity and medical image findings of patients with shoulder pain.

METHODS: This was an observational analytical study with 54 patients with possible shoulder injury. Participants have answered an incapacity questionnaire, a pain intensity scale and then were submitted to image exams. Incapacity, pain intensity and image diagnosis were correlated, in addition to comparing pain intensity and level of incapacity among participants with and without medical image abnormalities.

RESULTS: Mean pain intensity was $7.4 \pm 2.52$. There has been a high degree of incapacity (mean of $57.2 \%$ ). Image findings have shown abnormalities in $59.3 \%$ of reports. There has been no correlation between image findings and pain intensity, as well as there has also been no correlation between image findings and incapacity. There has been high correlation between pain intensity and incapacity (Rho=0.67; $\mathrm{p}<0.001)$. Pain intensity and incapacity were not different between participants with and without some type of abnormality at image exams.

CONCLUSION: Patients with shoulder complaints may have limitations to perform daily activities and pain, even with negative image findings.

Keywords: Deficit evaluation, Medical exams, Physical evaluation, Radiography, Shoulder pain.

1. Clínica Santa Helena, Cruzeiro, SP, Brasil.

2. Hospital Universitário Gaffrée e Guinle, Rio de Janeiro, RJ, Brasil.

3. Centro Municipal de Reabilitação do Rio de Janeiro, Rio de Janeiro, RJ, Brasil.

4. Centro Universitário Augusto Motta, Instituto Federal de Educaçâo Ciência e Tecnologia

do Rio de Janeiro, Rio de Janeiro, RJ, Brasil.

Apresentado em 30 de maio de 2014.

Aceito para publicação em 25 de agosto de 2014 .

Conflito de interesses: não há.

Endereço para correspondência:

Leandro Alberto Calazans Nogueira

Avenida Alfredo Balthazar da Silveira, 419 - bloco 01/apt ${ }^{\circ} 2308$

22790-710 Rio de Janeiro, RJ, Brasil.

E-mail: leandro.nogueira@ifrj.edu.br

(C) Sociedade Brasileira para o Estudo da Dor

\section{RESUMO}

JUSTIFICATIVA E OBJETIVOS: O quadro doloroso do ombro limita o paciente de realizar suas tarefas diárias, assim como de realizar atividades dentro da sua jornada de trabalho. $\mathrm{O}$ objetivo deste estudo foi avaliar a relação entre a intensidade da dor, a incapacidade e os achados no exame de imagem de pacientes com dor no ombro. MÉTODOS: Foi realizado um estudo observacional analítico em 54 pacientes com possível lesão de ombro. Os participantes responderam um questionário de incapacidade, uma escala de intensidade de dor e em seguida foram submetidos ao exame de imagem. Foi realizada a análise de correlação entre a incapacidade, a intensidade de dor e o diagnóstico por imagem, além da comparação da intensidade da dor e do nível de incapacidade entre os participantes com e sem anormalidade no exame de imagem.

RESULTADOS: A intensidade de dor média encontrada foi de $7,4 \pm 2,52$. Houve alto grau de incapacidade (média de 57,2\%). Nos exames de imagem analisados foram encontradas anormalidades em $59,3 \%$ dos laudos. Não houve correlação entre os achados na imagem e a intensidade de dor, assim como não houve correlação entre os achados na imagem e a incapacidade. Observou-se alta correlação entre intensidade de dor e o nível de incapacidade ( $R h o=0,67$; $\mathrm{p}<0,001)$. A intensidade de dor e incapacidade náo foram diferentes entre os participantes com ou sem algum tipo de anormalidade nos exames de imagem.

CONCLUSÁO: Pacientes com queixas na região do ombro podem apresentar limitaçóes nas atividades de vida diária e presença de dor, mesmo sem achados no exame de imagem.

Descritores: Avaliação da deficiência, Dor de ombro, Exame físico, Exames médicos, Radiografia.

\section{INTRODUÇÃO}

Queixas de dor e incapacidade da extremidade superior são relatos comuns na área de ortopedia. Estima-se que cerca de $20 \%$ da população geral apresenta dor crônica na extremidade superior ${ }^{1}$. Dentre essas condiçóes destacam-se os comprometimentos da regiáo do ombro. A prevalência de dor no ombro tem sido reportada entre 7 e $14 \%$ da população geral ${ }^{2}$. Os diagnósticos mais frequentes da região do ombro são: tendinopatia de manguito rotador, síndrome do impacto, distúrbio da articulaçáo acromio-clavicular e capsulite adesiva $^{3}$. 
Com a introdução da ultrassonografia, utilizando-se transdutores de alta resolução, a digitalização da radiografia, a modernidade da tomografia computadorizada e da ressonância nuclear magnética, ampliou-se muito a capacidade diagnóstica do exame de ombro e de outras áreas do sistema musculoesquelético. Apesar disso, a correlação entre os achados na imagem, a dor e a alteraçáo no exame físico é controversa ${ }^{4}$.

$\mathrm{O}$ exame físico do ombro apresenta padróes semelhantes de incapacidade independentemente do diagnóstico clínico. A redução da amplitude de movimento para elevação do ombro é um achado frequente em algumas condições, tais como: síndrome do impacto, tendinopatia do manguito rotador, bursite e capsulite adesiva. Em geral, a redução da elevação do ombro está associada às limitaçóes das atividades de vida diária. Estudo 5 encontrou mais de $70 \%$ da amostra com comprometimento para dormir confortavelmente no ombro afetado, lavar as costas no ombro oposto ao afetado, levantar 3,6kg e arremessar uma bola por cima da cabeça.

$\mathrm{O}$ quadro doloroso do ombro limita o paciente de realizar suas funçóes comuns do dia-a-dia, assim como de realizar atividades dentro da sua jornada de trabalho. Considerando essa associação, o presente estudo objetivou avaliar a relaçáo entre a intensidade da dor, a incapacidade e os achados no exame de imagem de pacientes com dor no ombro.

\section{MÉTODOS}

Foi realizado um estudo transversal observacional analítico em 54 pacientes com possível lesão de ombro na clínica de imagem Dr. Niazi Dias Rubez. Os participantes foram abordados no momento da realização do exame de imagem, independentemente de radiografia ou ultrassonografia. Os critérios de inclusão foram apresentar pedido de exame de imagem para a regiáo do ombro, com idade superior a 18 anos, independentemente do gênero. Foram excluídos os pacientes não colaborativos, com algum déficit cognitivo ou que tivessem cirurgia prévia de ombro.

Os participantes que concordaram em participar da pesquisa foram encaminhados para responder um questionário de incapacidade e uma escala de intensidade de dor. A coleta de dados foi realizada enquanto os pacientes aguardavam para a realização do exame de imagem. Após o preenchimento do questionário de incapacidade e da escala de dor, o paciente foi submetido ao exame regularmente.

A incapacidade da extremidade superior foi medida utilizando a versão curta do questionário autoaplicável Disabilities of the Arm, Shoulder and Hand (DASH). Esse questionário foi elaborado originariamente no idioma inglês e denominado DASH Questionnaire. O DASH foi desenvolvido por Hudak, Amadio e Bombardier ${ }^{6}$ com o objetivo de mensurar a incapacidade física e os sintomas dos membros superiores (MMSS) em uma população heterogênea. Orfale et al. ${ }^{7}$ traduziram e fizeram a adaptação transcultural do DASH para a língua portuguesa do Brasil. As variáveis foram analisadas através dos módulos propostos pelo DASH. A versão curta do questionário DASH tem 19 questóes (pontuadas de um a cinco). Os escores obtidos no QuickDash foram calculados da seguinte forma: [(soma das respostas / n) - 1] x 25, sendo que n é o número completo de respostas. $\mathrm{O}$ escore obtido quantifica o grau de incapacidade física dos MMSS do indivíduo. Quanto maior o resultado, maior a incapacidade. A pontuação é considerada como excelente ( $<20$ pontos), boa (20 a 39 pontos), regular (40 a 60 pontos) e má ( $>60$ pontos). As propriedades psicométricas da versão curta do questionário na língua portuguesa foram avaliadas por Puga et al. ${ }^{8}$ e a sua utilização é recomendada em pacientes com dor no ombro.

A intensidade de dor foi avaliada pela escala numérica de dor. A escala numérica de dor possui valores variando de zero (ausência de dor) a 10 (pior dor imaginável). Os participantes foram orientados a identificar o valor numérico que melhor identificava sua intensidade dolorosa na semana anterior.

Os exames de imagem realizados foram a radiografia e a ultrassonografia. As radiografias foram realizadas por um único técnico nas posiçóes anteroposterior (AP) corrigido e perfil de escápula em um aparelho digital (SHIMADZU MEDICAL). Tais incidências foram escolhidas por retratar de maneira clara as estruturas que envolvem essa articulação. De acordo com Turtelli ${ }^{9}$, a incidência AP corrigido é feita em anteroposterior, com rotação de $30^{\circ}$ do paciente para o lado do ombro examinado. Isso é feito para corrigir a anteversão da glenoide e a retroversão do úmero tangenciando a articulação em AP. A incidência de perfil da escápula ou incidência em Y, incidência do túnel ou desfiladeiro («outlet») foi feita com o paciente no mural (ereto), com o ombro a examinar rodado posteriormente em $10^{\circ}$, o suficiente para dissociar o ombro contralateral. $\mathrm{O}$ raio central incidia no acrômio inclinado $20^{\circ}$ caudal.

O exame de ultrassom foi realizado por um único médico experiente que não foi notificado previamente da utilizaçáo dos laudos, nas posiçóes flexáo do cotovelo a $90^{\circ}$ para avaliação do tendáo da cabeça longa do bíceps, rotação externa para avaliação do tendão do subescapular, rotação externa com extensão do braço (pedido ao paciente que colocasse o dorso da máo nas costas) para avaliação do tendão do supra espinhal, abdução com a mão na cintura para avaliação do tendão do infraespinhal, rotação interna com abdução horizontal (mão no ombro oposto) para avaliação da articulação acrômio-clavicular; em posição neutra foram observadas as bursas, assim como em abdução e adução.

A partir da análise dos laudos dos exames de imagem, os participantes foram classificados em três grupos: grupo 1 com estrutura anatômica preservada (normal), grupo 2 com sinais de síndrome do impacto (bursite, tendinopatia), ou grupo 3 com sinais de degeneração.

\section{Análise estatística}

A distribuição dos dados foi investigada pelo teste de Shapiro-Wilk. Foi realizada a análise de correlação entre a incapacidade, a intensidade de dor e o diagnóstico por imagem, utilizando o coeficiente de correlação de Pearson ou Spearman, de acordo com a natureza da distribuição dos dados. A correlação acima de 0,90 foi interpretada como muito alta $(0,70-0,89)$ alta $(0,50-0,69)$ moderada $(0,30-0,49)$ baixa e abaixo de 0,29 como discreta ${ }^{10}$. A amostra foi dicotomizada de acordo com o resultado do exame de imagem, sendo que um grupo foi classificado com exame normal e o outro grupo com sinais de impacto e/ou sinais degenerativos. Os grupos foram comparados pelo teste $\mathrm{T}$ e o teste $\mathrm{U}$ de Mann-Whitney, 
de acordo com a natureza da distribuição dos dados. O nível de significância adotado foi 0,05 para todos os testes estatísticos. O programa utilizado para a análise estatística foi o Statistical Package for Social Sciences(SPSS - versão 17).

Todos os participantes incluídos no estudo assinaram o Termo de Consentimento Livre e Esclarecido (TCLE). Este estudo foi conduzido de acordo com as diretrizes da resoluçáo 466/12, do Conselho Nacional de Saúde, em conformidade com a Declaração de Helsinque de 1975.

Este estudo foi aprovado sob o número CAAE 14939213.2.0000. $5258 / 2013$.

\section{RESULTADOS}

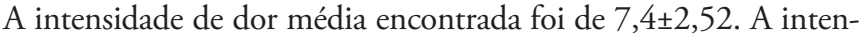
sidade de dor mais frequente (13 pacientes) foi o valor máximo (grau 10) e o segundo grau mais assinalado foi grau 5, correspondente a 8 pacientes. Houve alto grau de incapacidade (média de $57,2 \%$ ) medido pelo questionário QuickDASH, A maior parte das respostas foi assinalada como dificuldade moderada ou grave. As características da intensidade de dor, da incapacidade e do laudo do exame de imagem encontram-se na tabela 1 .

Tabela 1.Características da intensidade de dor, da incapacidade e do laudo do exame de imagem de pacientes com possível lesão de ombro $(n=54)$

\begin{tabular}{|c|c|}
\hline Características & Valores \\
\hline Incapacidade (QuickDash) (média $\pm D P)$ & $57,2 \pm 22,2$ \\
\hline Intensidade de dor (média $\pm \mathrm{DP}$ ) & $7,4 \pm 2,5$ \\
\hline \multicolumn{2}{|l|}{ Diagnóstico } \\
\hline Normal valor absoluto (\%) & $22(40,7)$ \\
\hline Sinais de impacto subacromial valor absoluto (\%) & $28(51,9)$ \\
\hline Sinais de degeneração valor absoluto (\%) & $4(7,4)$ \\
\hline
\end{tabular}

Nos exames de imagem analisados foram encontradas anormalidades em 32 (59,3\%) dos laudos. Em 25 laudos foram encontrados algum tipo de tendinopatia e em 7 sujeitos encontrou-se imagem compatível com bursite, as duas últimas que caracterizavam um sinal de síndrome do impacto, que supostamente são as maiores queixas nos atendimentos, tendinopatia e/ou bursite. A distribuição dos diagnósticos encontrada nos exames de imagens encontra-se na tabela 2.

A análise de correlação observou que não houve correlação entre os achados na imagem e a intensidade de dor ( $R h o=0,19 ; \mathrm{p}=0,89)$, assim como não houve correlaçáo entre os achados na imagem e a incapacidade ( $R h o=0,07 ; p=0,61)$. A única correlação estatisticamente significativa classificada como alta correlaçáo observada foi entre intensidade de dor e nível de incapacidade (Rho=0,67; $\mathrm{p}<0,001$ ) (Figura 1).

Os resultados de intensidade de dor e incapacidade não apresentaram diferença significativa entre os participantes que apresentaram ou não algum tipo de alteração nos exames de imagem. As figuras 2 e 3 apresentam a comparação entre a média de intensidade de dor e a média de incapacidade, respectivamente, nos grupos com imagem normal e imagem com lesão.
Tabela 2. Distribuição dos diagnósticos encontrados no exame de imagem de pacientes com possível lesão no ombro $(n=54)$

\begin{tabular}{lc}
\hline Resultados das radiografias e ultrassonografias & Valores \\
Laudo & 5 \\
\hline Tendinopatia do supraespinhal sem rotura & 12 \\
Tendinopatia do supraespinhal com rotura parcial & 3 \\
Tendinopatia do supraespinhal com rotura completa & 2 \\
Tendinopatia do manguito & 1 \\
Tendinopatia da cabeça longa do bíceps & 7 \\
Bursite subacromial/subdeltoideana & 1 \\
Rotura da cabeça longa do bíceps & \\
Tendão do supra discretamente espessado com calci- & 2 \\
ficação & 1 \\
Irregularidades dos contornos do tubérculo umeral & 1 \\
Processo inflamatório acrômio-clavicular & 1 \\
Sinais de osteoartrose acrômio-clavicular & 3 \\
Esporão acromial & 2 \\
Calcificação do subescapular & 1 \\
Lipoma & 22 \\
Normalidade &
\end{tabular}

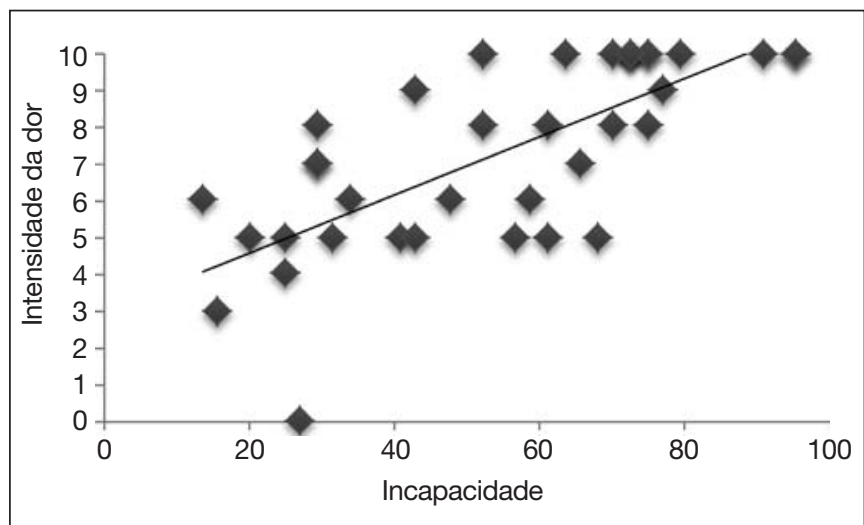

Figura 1. Correlação entre a intensidade de dor e a incapacidade em pacientes com possível lesão no ombro $(n=54)$

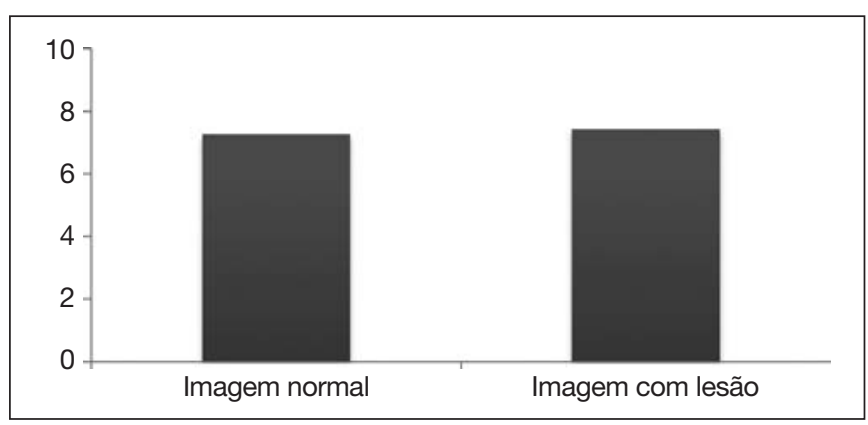

Figura 2. Comparação da intensidade de dor nos grupos com imagem normal e imagem com lesão em pacientes com possível lesão no ombro $(n=54)$ 


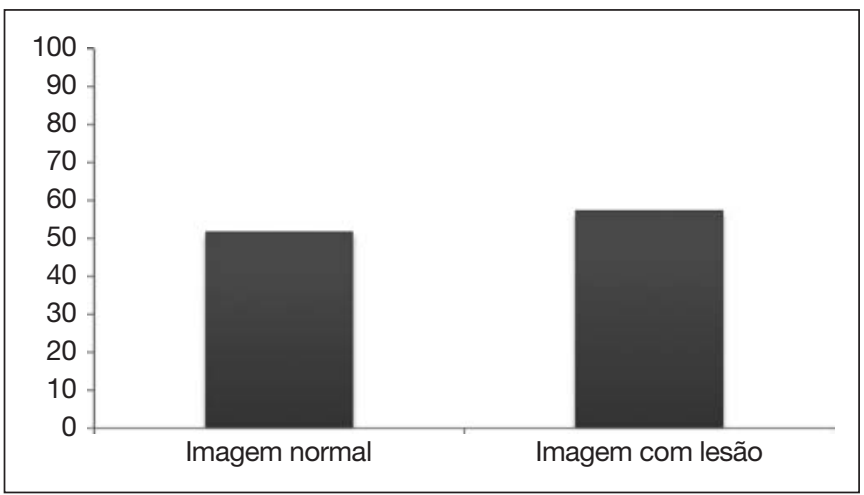

Figura 3. Comparação da incapacidade nos grupos com imagem normal e imagem com lesão em pacientes com possível lesão no ombro $(n=54)$

\section{DISCUSSÃO}

Os pacientes com queixas na região do ombro que foram encaminhados para o exame complementar com diagnóstico por imagem analisados no presente estudo apresentaram alta intensidade de dor, alto grau de incapacidade e diversos achados no exame de imagem. A intensidade de dor apresentou uma alta correlação com o nível de incapacidade. Apesar de vários pacientes apresentarem achados no exame de imagem, a lesão anatômica não esteve relacionada com a intensidade da dor nem com o grau de incapacidade.

As lesôes do manguito podem estar relacionadas com o processo de envelhecimento, uma vez que é mais provável encontrar uma anormalidade no exame de ressonância magnética em uma população idosa assintomática do que jovens com ombro doloroso sem tais lesóes ${ }^{11}$. Segundo Zorzetto et al..$^{12}$ aproximadamente $60 \%$ das alteraçôes do ombro estão ligadas a lesões do manguito rotador. Entretanto, apenas $10 \%$ das lesóes do manguito rotador podem apresentar alteração em sua morfologia, podendo obter retração do tendão, atrofia muscular e infiltraçóes ${ }^{13}$.

A comparação da intensidade de dor e do nível de incapacidade em pacientes com e sem lesão na imagem revelou não haver diferença nessas duas características entre os grupos. Um estudo prévio com utilização de ressonância magnética em pacientes com lesão de ombro demonstrou que embora achados de tendinopatia e bursite tivessem sido frequentes, a contribuição desses fatores para a sintomatologia foi baixa. Analisando a função, os mesmos autores encontraram resultados semelhantes àqueles aqui apresentados e revelaram que as atividades de vida diárias não foram afetadas mesmo em casos de rotura completa ou incompleta do manguito rotador ${ }^{11}$.

Hodges e Tucker $^{14}$ realizaram uma revisão da influência da dor na realização de tarefas com movimento e descreveram que o estímulo doloroso lidera uma modificação do comportamento muscular, do padrão de movimento e uma redução da velocidade do movimento além de outras repercussóes no sistema nervoso periférico e central. Com isso, a presença do quadro álgico deve explicar melhor as incapacidades apresentadas pelos pacientes do que os achados no exame de imagem.

Relatos na literatura corroboram nossos achados e apontam para o fato de que a lesão tecidual encontrada nos pacientes nem sempre reflete a condição funcional para realização de tarefas dinâmicas. Isto é, a gravidade da degeneração estrutural das articulaçôes e tecidos moles não está diretamente relacionada com o grau de alteraçáo funcional na maior parte dos pacientes com lesão musculoesquelética. Deve-se destacar ainda que os exames complementares em muitos casos não são relevantes e os achados radiológicos "falsos positivos" são cada vez mais comuns ${ }^{15}$.

Encontrar associação entre os sintomas e as imagens é uma tarefa desafiadora e essencial para a tomada de decisão clínica. Existem relatos de alta prevalência de lesôes do manguito rotador em população assintomática $^{16-18}$. Portanto, o uso indevido da imagem sem dados clínicos pode diminuir bastante a efetividade do tratamento e levar o profissional a escolhas inadequadas da intervenção terapêutica ${ }^{11}$. Devido à discrepância entre a presença de dor e incapacidade e os achados no exame de imagem, nas últimas décadas, desfechos como qualidade de vida relacionada a saúde, capacidade funcional, escalas de dor e satisfação do paciente têm sido utilizados como instrumentos de avaliação. Por possibilitarem a análise da situação de saúde e as manifestaçôes da doença na vida do indivíduo em sua própria perspectiva, tais avaliaçóes complementam os dados clínicos e objetivos como, por exemplo, amplitude de movimento, força muscular e os exames complementares ${ }^{19}$.

É sabido que o resultado do exame de imagem é dependente da habilidade do examinador na realização da técnica e a falta de precisão no laudo pode colaborar para algumas diferenças no resultado encontrado no presente estudo. A relaçáo entre a intensidade de dor e a incapacidade, que foi o principal achado do estudo, pode sofrer interferência de outros fatores, tais como: idade, gênero, ocupação, níveis de atividade física, padrão de movimento. Futuros estudos devem controlar esses fatores para analisar mais precisamente a relação aqui encontrada. Além disso, estudos com a utilização da ressonância magnética e com maior número de participantes também devem ser conduzidos para confirmar os achados apresentados no presente estudo.

\section{CONCLUSÃO}

Pacientes com queixas na região do ombro podem apresentar limitaçóes nas atividades de vida diária e presença de dor, mesmo sem achados no exame de imagem. A intensidade de dor e o nível de incapacidade não estáo relacionados com os achados do exame de imagem.

\section{REFERÊNCIAS}

1. Gummesson C, Atroshi I, Ekdahl C, Johnsson R, Ornstein E. Chronic upper extremity pain and co-occurring symptoms in a general population. Arthritis Rheum. 2003;49(5):697-702.

2. Bergenudd H, Lindgärde F, Nilsson B, Petersson CJ. Shoulder pain in middle age. A study of prevalence and relation to occupational work load and psychosocial factors. ClinOrthopRelat Res. 1988;(231):234-8.

3. Ostör AJ, Richards CA, Prevost AT, Speed CA, Hazleman BL. Diagnosis and relation to general health of shoulder disorders presenting to primary care. Rheumatology. 2005; $44(6): 800-5$

4. Maeda EY, HelfensteinJr M, Ascencio JE, Feldman D. O ombro em uma linha de produção: estudo clínico e ultrassonográfico. Rev Bras Reumatol. 2009;49(4):375-86.

5. Largacha M, Parsons IM 4th, Campbell B, Titelman RM, Smith KL, Matsen F 3rd. Deficits in shoulder function and general health associated with sixteen common shoulder diagnoses: a study of 2674 patients. J Shoulder Elbow Surg. 2006;15(1):30-9.

6. Hudak PL, Amadio PC, Bombardier C. Development of an upper extremity outcome measure: the DASH (disabilities of the arm, shoulder and hand) [corrected]. The Upper Extremity Collaborative Group (UECG). Am JIndMed. 1996;29(6):602-8. 
7. Orfale AG, Araújo PM, Ferraz MB, Natour J. Translation into Brazilian Portuguese, cultural adaptation and evaluation of the reliability of the Disabilities of the Arm, Shoulder and Hand Questionnaire. Braz JMedBiolRes.2005;38(2):293-302.

8. Puga VO, Lopes AD, Shiwa SR, Alouche SR, Costa LO. Clinimetric testing supports the use of 5 questionnaires adapted into Brazilian Portuguese for patients with shoulder disorders. J OrthopSports Phys Ther. 2013;43(6):404-13.

9. Turtelli CM. Avaliação do ombro doloroso pela radiologia convencional. Radiol Bras. 2001;34(4):241-6.

10. McDowell I. Measuring health: a guide to rating scales and questionnaires. $3^{\text {rd }}$ ed. New York: Press OU; 2006. 10-54p.

11. Krief OP, Huguet D. Shoulder pain and disability: comparison with MR findings. AJR Am JRoentgenol. 2006;186(5):1234-9.

12. Zorzetto AA, Urban LA, Liu CB, Prevedello L, Zapparoli M, Vitola ML, et al. A ecografia no diagnóstico das lesôes músculo-tendinosas do ombro. Radiol Bras. 2003;36(4):237-42.

13. Shahabpour M, Kichouh M, Laridon E, Gielen JL, De Mey J. The effectiveness of diagnostic imaging methods for the assessment of soft tissue and articular disorders of the shoulder and elbow.EurJRadiol. 2008;65(2):194-200.

14. Hodges PW, Tucker K. Moving differently in pain: a new theory to explain the adaptation to pain. Pain. 2011;152(3 Suppl):S90-8.

15. Flynn TW, Smith B, Chou R. Appropriate use of diagnostic imaging in low back pain a reminder that unnecessary imaging may do as much harm as good. JOrthopSports Phys Ther. 2011;41(11):838-46.

16. Sher JS, Uribe JW, Posada A, Murphy BJ, Zlatkin MB. Abnormal findings on magnetic resonance images of asymptomatic shoulders. J Bone Joint Surg Am. 1995;77(1):10-5.

17. Needell SD, Zlatkin MB, Sher JS, Murphy BJ, Uribe JW. MR imaging of the rotator cuff: peritendinous and bone abnormalities in an asymptomatic population. AJR Am JRoentgenol. 1996;166(4):863-7.

18. Tempelhof S, Rupp S, Seil R. Age-related prevalence of rotator cuff tears in asymptomatic shoulders. J Shoulder Elbow Surg. 1999;8(4):296-9.

19. Lopes AD, Ciconelli RM, Reis FB. Medidas de avaliação de qualidade de vida e estados de saúde em ortopedia. RevBras Ortop. 2007;42(11/12):355-9. 